\title{
THE HARDY CLASS OF A SPIRAL-LIKE FUNCTION AND ITS DERIVATIVE
}

\author{
T. BAŞGÖZE AND F. R. KEOGH ${ }^{1}$
}

Abstract. A determination is made of the Hardy classes to which a spiral-like univalent function and its derivative belong. An estimate for the size of the Taylor coefficients is deduced.

Let $f(z)$ be analytic in $D$, the unit disc $|z|<1$, and let $\alpha$ be a real number such that $|\alpha|<\pi / 2$. If $f(0)=0, f^{\prime}(0) \neq 0$, and if

$$
\operatorname{Re}\left[e^{i \alpha} z f^{\prime}(z) / f(z)\right]>0, \quad z \in D,
$$

then $f(z)$ is univalent [5] and is said to be spiral-like. Under these conditions we have

$$
e^{i \alpha} z f^{\prime}(z) / f(z)=Q(z),
$$

where $\operatorname{Re} Q(z)>0$ and $Q(0)=e^{i \alpha}$. Defining $P(z)=Q(z) \sec \alpha-i \tan \alpha$, we may write

$$
z f^{\prime}(z) / f(z)=e^{-i \alpha}[P(z) \cos \alpha+i \sin \alpha],
$$

where $\operatorname{Re} P(z)>0, P(0)=1$.

If $f^{\prime}(0)=1,{ }^{2}$ if $f(z)$ satisfies (1), and if $\operatorname{Re} P(z) \geqq \rho \geqq 0, z \in D$, we shall say that $f(z)$ belongs to the class $S_{\alpha, \rho}$ [3]. In particular, with $\alpha=0, S_{0, \rho}$ coincides with the class of normalized starlike functions of order $\rho$. The relationship between $S_{\alpha, \rho}$ and $S_{0, \rho}$ is indicated in the following lemma.

LeMma 1. $f(z) \in S_{\alpha, \rho}$ if and only if there is a $g(z) \in S_{0, p}$ such that

$$
[f(z) / z]^{\exp (i \alpha)}=[g(z) / z]^{\cos \alpha},
$$

where the branches are chosen so that each side of the equation has the value 1 when $z=0$.

PRoof. If $g(z) \in S_{0, \rho}$ then $z g^{\prime}(z) / g(z)=P(z)$, where $\operatorname{Re} P(z) \geqq \rho$, $P(0)=1$. If $f(z)$ is defined by (2) then, differentiating logarithmically and multiplying by $z e^{-i \alpha}$, we obtain (1), which shows that $f(z) \in S_{\alpha, \rho}$.

Received by the editors December 5, 1969.

AMS subject classifications. Primary 3040, 3042; Secondary 3067.

Key words and phrases. Hardy class, spiral-like univalent function, Hölder's inequality.

1 Research supported by NSF Grant GP-7377.

2 This normalization is made only for convenience. The conclusion of Theorem 2 and its corollary remain valid for the classes $S_{\alpha, \rho}$, when defined without normalization. 
Conversely, if $f(z) \in S_{\alpha, \rho}$ and if we define $g(z)$ by

$$
g(z) / z=[f(z) / z]^{1+i \tan \alpha},
$$

then a similar calculation shows that $g(z) \in S_{0, \rho}$.

For a real $\lambda>0$, we say that a function $h(z)$, analytic in $D$, belongs to the class $H^{\lambda}$ if

$$
\int_{-\pi}^{\pi}\left|h\left(r e^{i \theta}\right)\right|^{\lambda} d \theta<K
$$

for $0 \leqq r<1$, where $K$ is a constant depending on $h(z)$ and $\lambda$.

The following theorem is equivalent to Theorem 6 in [1].

THEOREM 1. If $g(z) \in S_{0, \rho}$ is not of the form

$$
g(z)=z\left(1-z e^{i \tau}\right)^{2 \rho-2}
$$

for some real $\tau$, then

(i) there exists $\delta=\delta(g)>0$ such that $g(z) / z \in H^{(1+\delta) / 2(1-p)}$; and

(ii) there exists $\epsilon=\epsilon(g)>0$ such that $g^{\prime}(z) \in H^{(1+\epsilon) /(3-2 \rho)}$.

The object of this note is to extend this theorem to the classes $S_{\alpha, \rho}$. To do this we require some further lemmas.

Lемма 2. If $g(z) \in S_{0,0}$, then

$$
|\arg (g(z) / z)|<\pi, \quad z \in D,
$$

where the principal value of the argument is taken.

Lemma 3. If $Q(z)$ is analytic and $\operatorname{Re} Q(z)>0$ in $D$, then $Q(z) \in H^{\lambda}$ for all $\lambda<1$.

LEMMA 4. If $h(z) \in H^{\lambda}, 0<\lambda<1$, and $h(z)=\sum_{0}^{\infty} a_{n} z^{n}$, then

$$
a_{n}=o\left(n^{(1 / \lambda)-1}\right) \text {. }
$$

Lemma 2 is in [4], Lemma 4 in [2]. Lemma 3 is well known.

The following theorem contains Theorem 1.

ThEOREM 2. If $f(z) \in S_{\alpha, \rho}$ is not of the form

$$
f(z)=z\left(1-z e^{i \tau}\right)^{-a}, \quad a=2(1-\rho)(\cos \alpha-i \sin \alpha) \cos \alpha
$$

for some real $\tau$, then

(i) there exists $\delta=\delta(f)>0$ such that

$$
f(z) / z \in H^{\mu}, \quad \mu=(1+\delta) \sec ^{2} \alpha / 2(1-\rho) ;
$$

and 
(ii) there exists $\epsilon=\epsilon(f)>0$ such that

$$
f^{\prime}(z) \in H^{\nu}, \quad \nu=(1+\epsilon) /\left(1+2(1-\rho) \cos ^{2} \alpha\right) .
$$

Proof. (i) By Lemma 1, there is a function $g(z) \in S_{0, \rho}$ such that

$$
f(z) / z=[g(z) / z]^{\cos ^{2} \alpha-i \sin \alpha \cos \alpha .}
$$

Taking moduli we obtain

$$
|f(z) / z|=|g(z) / z|^{\cos ^{2} \alpha} \exp [\sin \alpha \cos \alpha \arg (g(z) / z)],
$$

so

$|f(z) / z|^{\mu}=|g(z) / z|^{(1+\delta) / 2(1-\rho)} \exp [(1+\delta) \tan \alpha / 2(1-\rho) \arg (g(z) / z)]$.

By Lemma 2, since $S_{0, \rho} \subset S_{0,0}$, the exponential factor is bounded, and the conclusion follows immediately from part (i) of Theorem 1 .

(ii) Writing $e^{i \alpha} z f^{\prime}(z) / f(z)=Q(z)$, and applying Lemma 3 , we see that

$$
z f^{\prime}(z) / f(z) \in H^{\lambda}, \quad \text { all } \lambda<1 \text {. }
$$

Next, writing

$$
f^{\prime}(z)=(f(z) / z)\left(z f^{\prime}(z) / f(z)\right),
$$

and apply Hölder's inequality with conjugate indices $p, q$ to $\left|f^{\prime}(z)\right|^{\lambda}$, with $z=r e^{i \theta}$ we obtain

$$
\int_{-\pi}^{\pi}\left|f^{\prime}(z)\right|^{\lambda} d \theta \leqq\left(\int_{-\pi}^{\pi}\left|\frac{f(z)}{z}\right|^{\lambda p} d \theta\right)^{1 / p}\left(\int_{-\pi}^{\pi}\left|\frac{z f^{\prime}(z)}{f(z)}\right|^{\lambda q} d \theta\right)^{1 / q}=I_{1} \cdot I_{2},
$$

say. By part (i), $I_{1}$ is bounded if we choose $\lambda$, $p$ so that $\lambda p=\mu$, and by (4), $I_{2}$ is bounded if we make the further restriction $\lambda q<1$. This is achieved by taking $\lambda=\nu$ provided that

$$
\nu=\frac{1+\epsilon}{1+2(1-\rho) \cos ^{2} \alpha}<\frac{1+\delta}{1+\delta+2(1-\rho) \cos ^{2} \alpha},
$$

which holds if $\epsilon$ is sufficiently small.

From part (ii) of the theorem and Lemma 4 we deduce the following : if

COROLLARY. If $f(z) \in S_{\alpha, p}$ is not of the form (3) for some real $\tau$, and

$$
f(z)=\sum_{0}^{\infty} a_{n} z^{n}
$$

then there exists $\eta=\eta(f)>0$ such that 


$$
a_{n}=o\left(n^{2(1-\rho) \cos ^{2} \alpha-1-\eta}\right) .
$$

In conclusion we remark that for the function (3) we have

$$
\begin{gathered}
a_{n}=\frac{\Gamma(a+n)}{\Gamma(n+1) \Gamma(a)} e^{n i \tau} \\
\left|a_{n}\right| \sim\left|n^{a-1}\right| /|\Gamma(a)|=n^{2(1-\rho) \cos ^{2} \alpha-1} /|\Gamma(a)| .
\end{gathered}
$$

This shows that (3) is indeed exceptional in the corollary, and therefore also in part (ii) of Theorem 2; a computation shows also that for the function (3) we have $f(z) / z \in H^{\lambda}$ if and only if $\lambda<\frac{1}{2} \sec ^{2} \alpha /(1-\rho)$, so that this function is also exceptional in part (i) of Theorem 2.

A weaker consequence of the corollary, well known for starlike functions, is that for all spiral-like functions $f(z)$ with the exception only of those of the form $z\left(1-z e^{i \tau}\right)^{-2}$, we have $a_{n}=o\left(n^{1-\eta}\right)$ for some $\eta=\eta(f)>0$.

ADDED IN PROOF (June 16, 1970). Application of Theorem 2 and of Theorem $E$ of [1] yields the following result for functions of the class $C_{\alpha}[6]$.

If $f(z) \in C_{\alpha}$ is not of the form

$$
f(z)=e^{-i \pi}\left(1-z e^{i \pi}\right)^{-a+1} /(a-1)+c, \quad a=2(\cos \alpha-i \sin \alpha) \cos \alpha
$$

for some complex $c$ and real $\tau$, then there exists $\delta=\delta(f)>0$ such that

(i) $f^{\prime}(z) \in H^{\beta}, \beta=\frac{1}{2}(1+\delta) \sec ^{2} \alpha$;

(ii) if $|\alpha|<\pi / 4$ then $f(z) \in H^{\gamma}, \gamma=(1+\delta) \sec ^{2} \alpha /\left[2-(1+\delta) \sec ^{2} \alpha\right]$.

\section{REFERENCES}

1. Paul J. Eenigenburg and F. R. Keogh, On the Hardy class of some univalent functions and their derivatives, Michigan Math. J. (to appear).

2. G. H. Hardy and J. E. Littlewood, Some properties of fractional integrals. II, Math. Z. 34 (1932), 403-439.

3. R. J. Libera, Univalent $\alpha$-spiral functions, Canad. J. Math. 19 (1967), 449-456. MR 35 \#5599.

4. B. Pinchuk, On starlike and convex functions of order $\alpha$, Duke Math. J. 35 (1968), 721-734. MR 37 \#6454.

5. L. Špaček, Contribution d la theorie des fonctions univalentes, Casopis. Pešt. Mat. 62 (1933), 12-19.

6. M. S. Robertson, Univalent-functions $f(z)$ for which $z f^{\prime}(z)$ is spirallike, Michigan Math. J. 16 (1969), 97-101. MR 39 \#5785.

Middle East Technical University, Ankara, Turkey

University of KentuCKy, LeXington, Kentucky 40506 